Fakultät III

Wirtschaftswissenschaften, Wirtschaftsinformatik und Wirtschaftsrecht

Volkswirtschaftliche Diskussionsbeiträge

Discussion Papers in Economics

No. 157-12

November 2012

Moritz Bonn

Migrants 'Acquisition of Cultural Skills and Selective Immigration Policies 
Universität Siegen

Fakultät III

Wirtschaftswissenschaften, Wirtschaftsinformatik und Wirtschaftsrecht

Fachgebiet Volkswirtschaftslehre

Hölderlinstraße 3

D-57068 Siegen

Germany

http://www.wiwi.uni-siegen.de/vwl/

ISSN 1869-0211

Available for free from the University of Siegen website at

http://www.wiwi.uni-siegen.de/vwl/research/diskussionsbeitraege/

Discussion Papers in Economics of the University of Siegen are indexed in RePEc and can be downloaded free of charge from the following website:

http://ideas.repec.org/s/sie/siegen.html 


\title{
Migrants' Acquisition of Cultural Skills and Selective Immigration Policies
}

\author{
Moritz Bonn*
}

November 19, 2012

\begin{abstract}
Based on the requirement of OECD countries to permit substantial inflows of immigrants to compensate for the effects of the demographic change, this paper explores the incentives of heterogeneous migrants to acquire host country specific cultural skills to improve their labor market outcomes. The theoretical results predict that the migrants' ambition in achieving such skills is increased if the scope of their respective cultural group is small, social permeability of migrants in the native society is large and individual integration costs are low.

Based on these results, I study whether cultural heterogeneity among the migrant population is welfare enhancing for the native population. I find that as long as migrants do not differ too much with regard to their costs of learning the native culture, cultural heterogeneity is beneficial for the host economy. The model provides an explanation for the shift in the immigration policies of the traditional host countries throughout the twentieth century as well as the current immigration policies in the EU member states.
\end{abstract}

JEL Classification: F22 · J15 - O31

Keywords: Immigration, Cultural Interaction, Political Economy

*University of Siegen, Department of Economics, Hoelderlinstraße 3, 57068 Siegen, Germany.

Phone +49 271-740-4046, Fax +49 271-740 -4042,

e-mail: bonn@vwl.wiwi.uni-siegen.de 


\section{Introduction}

It has been long well-known that the industrialized countries will face a sharp decline in population size due to low fertility rates. More precisely, scientists anticipate that without immigration, the population of the developed countries will decline from 1.23 billion in 2009 to 1.15 billion in 2050 (Hugo 2011). At the same time, it is estimated that developing countries will experience a massive increase in population size (United Nations 2009) 1 The development is challenging for policy makers in several ways since a sufficiently large stock of young workers is required for funding social security and old age pension systems, prevents skill shortages, ensures creativity and fosters innovation.

Steady immigration is often regarded to be an adequate solution to compensate for the shrinkage of the "native" workforce. Hence, many countries are going to restructure their guidelines to facilitate the inflow and naturalization of suitable applicants from developing countries. At the same time, however, there is evidence that even skilled migrants are often insufficiently integrated into the native society and underperform the average native-born in job-related characteristics such as language skills, wages and employment rates (Chiswick and Miller 1998; Hatton and Williamson 2005). For instance, Chaloff and Lemaitre (2009: 39) report that according to a study covering 9 OECD countries, only $55 \%$ of high skilled foreign borns work in high skilled occupations, whereas this applies to $70 \%$ of native high skilled. On the other side, $26 \%$ of the high skilled foreign born population were unemployed or not in the labor force far above the corresponding $14 \%$ of native high skilled.

In this context, the migrants' acquisition of cultural skills such as language proficiency, knowledge on the host countries legal system, norms and taboos have become key issues for researchers and policy makers since they are assumed to improve the labor market perspectives. These are further counteracting anti-immigrant sentiments which are largely aggravating the required immigration flows. In recent years, much scientific effort has been spent on the determinants that help to explain the incentives that motivate and the obstacles that discourage migrants from achieving host country specific cultural skills.

This paper connects two important strings of the research on immigration and cultural integration. First, I present a simple model that investigates the cultural learning decision of migrants. In this context, I interpret cultural learning as a synonym for the achievement of country specific skills such as language proficiency or the knowledge of the legal system, cultural taboos and norms of the native population. Migrants face costs of learning the other

\footnotetext{
${ }^{1}$ For the UK it was estimated that an annual net inflow of 190,000 workers over a period of 75 years is needed to achieve an old age dependency ratio to 0.396 in 2081 whereas it was projected to be 0.431 if net immigration was 0 (Rowthorn 2008).
} 
culture but also achieve benefits since their ambition is rewarded on the labor market. The results achieved by the model are in line with the main findings of empirical works on this topic: Immigrants' acquisition of cultural skills is enhanced if they belong to a small cultural and linguistic minority, if permeability on the local labor market is large and if their ex ante costs of learning the new culture are comparably low (Chiswick and Miller 1995, 2002). The latter is generally the case if the traditional and the newly-learned culture are relatively similar 2 Second, based on these results, the paper further provides a simple model of how a native welfare maximizing government is going to design an immigration policy. The idea is that the migrants' achievement of cultural skills constitutes a positive externality for the native population since it facilitates communication and thus intercultural interaction. By use of an adequate selective immigration policy, the government can improve the migrants' achievement of host country specific cultural skills.

Instead of focusing on the entire scope of migrants which I suppose to be exogenously determined by factors such as a constant skill shortage caused by demographic change, I concentrate on their cultural composition. The government can either conduct a policy that favors enlarged cultural homogeneity among migrants or promote multicultural immigration. In fact, the responsible policy maker is involved in a trade off. If migrants, according to their country of origin, have asymmetric learning costs, it has incentives to restrict immigration to individuals from culturally proximate countries since they probably have less obstacles in achieving language skills or building relations to the native population. However, as empirical results indicate, the scope and concentration of immigrant groups also matter so that, given a constant need for migrants, cultural heterogeneity ceteris paribus improves integration of migrants.

The results of the model suggest that the government is going to pursue an immigration policy which promotes homogenous immigration as long as the learning costs of potential migrants from different source countries are very asymmetric. A reduction of these asymmetries will foster increased cultural diversity among migrants. At least to some degree, the model can explain the shift in the immigration policies of traditional host countries like those of the U.S. and Australia in the 1970s. Additionally, it provides some explanation for the differences between the immigration policies of these countries and that of the EU member states.

Up to now, research on cultural learning of migrants has predominantly been concentrated on empirical studies. Based on the pioneering work of Chiswick (1978), there has been a vast

\footnotetext{
${ }^{2}$ As an adequate example, Chiswick and Miller (1995) stated that the costs of learning the Spanish language largely depend on the individual's country of origin, for instance whether the individual is Italian or Korean.
} 
literature analyzing how well migrants integrate into the host country (predominantly the U.S.) and whether investments in cultural skills, such as language proficiency, leads to higher wages of migrants. Even though many empirical economists have contributed to this field of research, it is surprising that related theoretical work is hardly found. Two exceptions are Church and King (1993) and Lazear (1999) who respectively provide a simple model of language acquisition. Their frameworks are able to explain why relative population size of the own linguistic group negatively affects the individual investment in language skills. With regard to immigration policy, Lazear (1999) therefore concludes that assimilation into the native culture is more probable if the scope of immigrants is relatively small $]^{3}$

Lazear (1999) further points at the importance of a neutral or dominant culture in multicultural societies which, similar to an overall accepted currency, facilitates trade between individuals of different cultural origin. In this paper, I basically regard the same issue from a reverse angle: Given that a dominant (native) culture exists, what is the most favorable cultural composition of migrants from the host country's point of view? Is a heterogeneous or homogenous immigrant population optimal to encourage migrants to acquire cultural skills? This issue is important, as one is aware of the circumstance that throughout the 20th century immigration guidelines had often been constructed in a way that they restricted access to the host country to applicants from specific culturally close source countries. For instance, immigration from Asia into the U.S. and Australia had been largely restricted until the 1970s (Green1995; Hatton and Williamson 2005; Leuner 2007).

This paper is structured as follows: the next section investigates the determinants that influence immigrants to achieve cultural skills. Based on this, Section 3 studies the government's choice for an optimal composition of migrants. Section 4 extends the framework by distinguishing intra- and inter-group heterogeneity with respect to the migrants' original cultures. Section 5 compares the basic results to some developments in reality. Section 6 concludes.

\section{The Acquisition of Cultural Skills}

In this section, I model the acquisition of host country specific cultural skills of migrants with different cultural origin. There are three potential types of individuals in the economy: natives and two types of immigrants. Let the total population be normalized to unity. The

\footnotetext{
${ }^{3}$ However, Lazear (1999) also states that increased cultural diversity may also enhance innovation and thus be beneficial for the host economy. Bonn (2012) has recently taken account of this circumstance and implemented a cultural gain parameter in a model framework similar to the one provided by Lazear (1999) in order to show that moderate immigration rates might be favorable policy outcomes .
} 
stock of all migrants is equal to $m$, where $m<0.5$ so that the native population denoted by $1-m$ constitutes the majority. Every group is characterized by an own specific culture, however the native population represents the dominant culture. This applies to the official language, norms and rights. In this section, the size of each cultural group is exogenous and within each cultural group, members are assumed to be homogenous. Suppose that individuals are interested in maximizing their labor income.

Individual income depends on the ability to interact with other individuals which is determined by their respective competence to communicate effectively with each other. The communication skills require cultural knowledge. This can be well understood if one takes into account that language proficiency of migrants significantly improve their earning perspectives (Chiswick and Miller 1995, 1999).

Basic assumptions of the model are similar to the model provided by Lazear (1999). I suppose that a representative migrant from country $i=1,2$ has the following expected payoff function:

$$
E W_{M_{i}}=\bar{w}\left(X_{M_{i}}-C L_{M_{i}}\right)
$$

where $\bar{w}$ is a constant wage factor which describes the wage level of the economy. $X_{M_{i}}$ describes the migrant's productivity level which depends on the ability to communicate with other individuals. Investments in cultural skills such as language proficiency increase $X_{M_{i}}$, but also lead to costs $C L_{M_{i}}$ since time that is used for learning reduces the potential working time.

Suppose that the migrants' productivity equals the expected payoffs which are achieved by interaction with other individuals as described by (2):

$$
X_{M_{i}}=E Y_{M_{i}}=P\left(M_{i}, N\right) Y_{M_{i}, N}+P\left(M_{i}, M_{i}\right) Y_{M_{i}, M_{i}}+P\left(M_{i}, M_{j \neq i}\right) Y_{M_{i}, M_{j \neq i}}
$$

$Y_{M_{i}, N}, Y_{M_{i}, M_{i}}$ and $Y_{M_{i}, M_{j \neq i}}$, respectively, depict the alternative payoffs which the migrant receives if he interacts with a native, a migrant of the same origin or a migrant of the other origin. $P\left(M_{i}, N\right), P\left(M_{i}, M_{i}\right)$ and $P\left(M_{i}, M_{j \neq i}\right)$ define the corresponding probabilities that these interactions take place. Individuals match pairwise, and matching is partly random. More precisely, I suppose that to a certain share $\alpha$, an individual will always encounter an individual of the own cultural community. With probability $(1-\alpha)$ the individual is 
matched totally randomly. Hence, $\alpha$ indicates the scope of cultural segregation. Similar to Burchardi (2011), I assume that the scope of cultural segregation is exogenous from the individual point of view. The idea is that, independent of further individual characteristics, immigrants are highly concentrated in certain occupations and often live together in specific areas. Probably, cultural ties and traditional norms prevent interactions between individuals of different cultural communities. Thus, the probabilities that a migrant from country 1 interacts with a native $P\left(M_{1}, N\right)$, an immigrant of the same cultural group $P\left(M_{1}, M_{1}\right)$ and an immigrant from country $2 P\left(M_{1}, M_{2}\right)$ are:

$$
\begin{gathered}
P\left(M_{1}, N\right)=(1-\alpha)(1-m), \\
P\left(M_{1}, M_{1}\right)=\alpha+(1-\alpha)(1-\beta) m, \\
P\left(M_{1}, M_{2}\right)=(1-\alpha) \beta m
\end{gathered}
$$

where $\beta$ accounts for the share of migrants from country 2. In other words, the matching probabilities depend on the degree of segregation $\alpha$ as well as on the population size of the respective cultural group which is determined by $m$ and $\beta$. As mentioned, the payoffs which are achieved by interaction depend on the ability of the encountering individuals to communicate with each other. If migrants interact with a member of the same cultural community, they receive a reference payoff $Y_{M_{i}, M_{i}}=1$. If the matching individuals have different cultural origins, their payoff depends on their cultural skills. Assume that an immigrant from country $i=1,2$ achieves the following outcome if interaction takes place with a native individual:

$$
Y_{M_{i}, N}=\psi \phi_{i}
$$

where $\phi_{i}$ describes the migrant's knowledge of the domestic culture. The variable thus describes the migrants' degree of cultural skills. $\psi \geq 0$ points at the various sets of asymmetries between the job opportunities of the native and the migrant population. Beside language and other cultural skills, immigrants are often disadvantaged in achieving high paid jobs since they have a lack of specific knowledge and limited access to business networks (Hatton and Williamson 2005: 314). Interaction with the native population may reduce these deficits and improve the migrant's career perspectives. This is reasonable if the vast majority of firms and institutions are run by natives. On the other hand, interaction with natives may bear some disadvantages for the migrant. This can be due to cultural or ethnical discrimination or due to the non-acceptance of qualifications which were achieved in the migrant's home country. A 
large $\psi>1$ indicates that interaction with the dominant culture is ceteris paribus positive for the migrant, meaning that the positive effects outweigh the negative ones. It is possible that $\psi$ varies between countries indicating that labor market outcomes for immigrants depend on the structure of the respective host economy (Hatton and Williamson 2005: 323).

Suppose that interaction between immigrants of different cultural origins may only take place by use of the dominant culture. E.g. consider that immigrants from Mexico and China need to communicate with each other in English after they immigrated into the USA. Thus, their potential benefits not only depend on the own language proficiency but also on that of the encountered individual. Accordingly, an immigrant from country $i=1,2$ achieves the following payoff if he interacts with an immigrant from country $j \neq i$ :

$$
Y_{M_{i}, M_{j \neq i}}=\phi_{i} \phi_{j \neq i}
$$

Equation (7) indicates that cultural knowledge is a positive externality for migrants of other cultural origins. The degree of cultural learning is not an exogenous parameter but depends on the individual ambition to learn the official culture. Consider for instance that an immigrant needs to trade off the benefit of enlarged language skills with the costs which arise in terms of time that is spent on learning and therefore cannot be used for work. In order to simplify the model, I assume that the time costs of enlarged cultural learning are quadratic. E.g. basic language skills are easily learned whereas absolute proficiency in speaking a language can only be reached by intensive study. Suppose further that immigrants from country 1 are advantaged in the way that their original culture is closer to the native one so that they face lower learning costs than migrants from country 2. By assuming that, ceteris paribus, $\lambda$ accounts for the relative learning cost mark-up of individuals from country 2 , the learning costs for an immigrant from country 1 and 2 are $C L_{M_{1}}=\frac{\theta_{1}^{2}}{2}$ and $C L_{M_{2}}=(1+\lambda) \frac{\theta_{2}^{2}}{2}$. Inserting into (1) leads to the following expected payoff functions for migrants from country 1 and 2.

$$
\begin{gathered}
E W_{M_{1}}=\bar{w}\left((1-m)(1-\alpha) \psi \phi_{1}+\alpha+(1-\alpha)(1-\beta) m+\beta m(1-\alpha) \phi_{1} \phi_{2}-\frac{\theta_{1}^{2}}{2}\right) \\
E W_{M_{2}}=\bar{w}\left((1-m)(1-\alpha) \psi \phi_{2}+\alpha+(1-\alpha) \beta m+(1-\beta) m(1-\alpha) \phi_{1} \phi_{2}-(1+\lambda) \frac{\theta_{1}^{2}}{2}\right)
\end{gathered}
$$

By maximizing (8) and (9) with respect to $\phi_{1}$ and $\phi_{2}$ one achieves the following optimal 


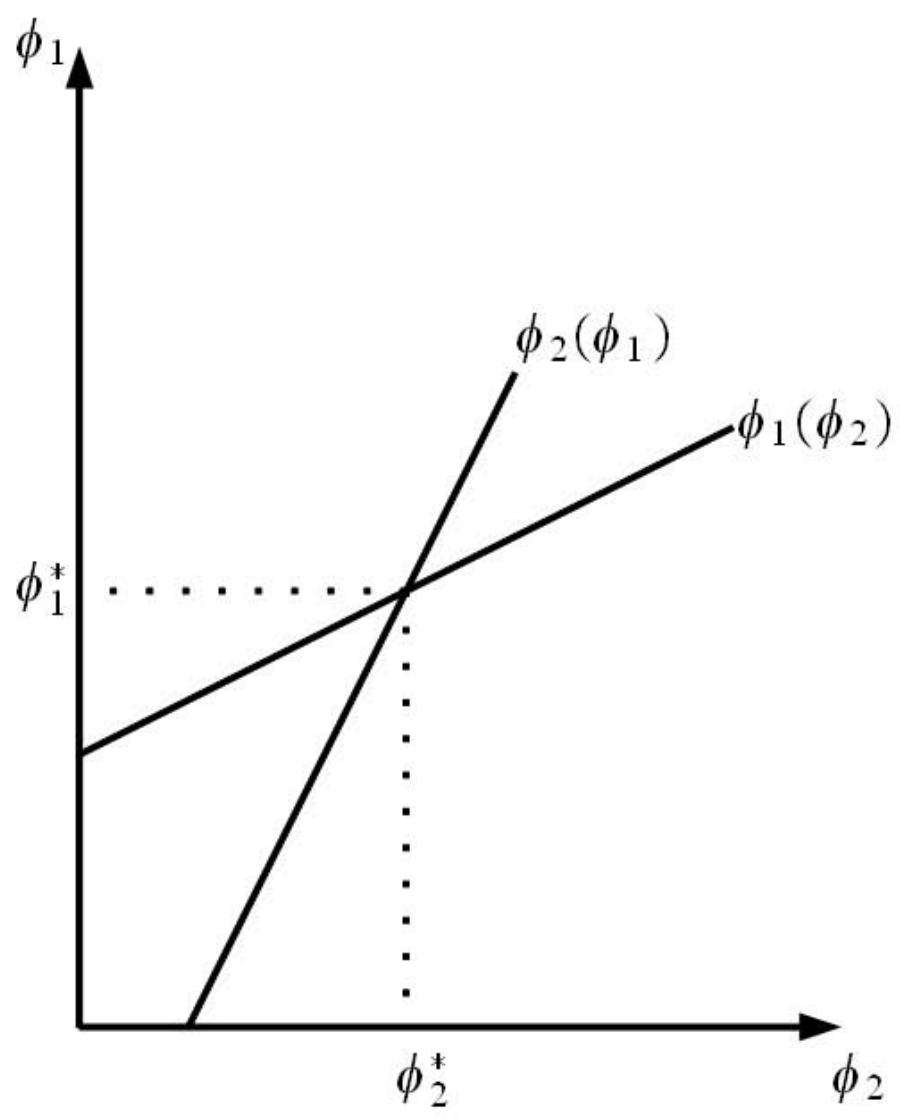

Figure 1: Nash equilibrium

response functions for migrants from country 1 and 2 :

$$
\begin{gathered}
\phi_{1}\left(\phi_{2}\right)=(1-\alpha)(1-m) \psi+(1-\alpha) \beta m \phi_{2}, \\
\phi_{2}\left(\phi_{1}\right)=\frac{(1-\alpha)(1-m) \psi+(1-\alpha)(1-\beta) m \phi_{1}}{1+\lambda} .
\end{gathered}
$$

(10) and (11) indicate that cultural integration of one immigrant population positively affects the integration behavior of the other immigrant group. One can interpret both curves as two Nash reaction functions with positive slope as illustrated by figure 1.

The equilibrium levels of cultural integration of migrants from country 1 and 2 are determined by the intersection point of (10) and (11).

$$
\phi_{1}^{*}=\frac{(1-\alpha)(1-m) \psi(1+\lambda+m \beta(1-\alpha))}{1+\lambda-\beta(1-\beta)(1-\alpha)^{2} m^{2}}
$$




$$
\phi_{2}^{*}=\frac{(1-\alpha)(1-m) \psi(1+m(1-\beta)(1-\alpha))}{1+\lambda-\beta(1-\beta)(1-\alpha)^{2} m^{2}}
$$

From (12) and (13) one can easily deduce that an increase of the permeability of the national labor market indicated by $\psi$ has a positive effect, whereas an increase in cultural segregation described by parameter $\alpha$ has a negative effect on cultural learning of both immigrant groups $4^{4}$ An increase of $\lambda$ will decrease cultural knowledge of both types of immigrants even though only immigrants from country 2 are directly affected by it. This can be explained by the mentioned externality between cultural learning of migrants of different cultural origins. The costs that prevent one type of migrants to achieve proficiency in the official language for instance indirectly influence the learning ambition of other immigrant groups. The size of the total immigrant population $m$ decreases the learning ambition of migrants. Differentiations of (12) and (13) show that

$$
\begin{aligned}
\frac{\partial \phi_{1}^{*}}{\partial m}= & -(1-\alpha) \psi \frac{(1+\lambda-\beta(1-\alpha))\left(1+\lambda+\beta(1-\beta)(1-\alpha)^{2} m^{2}\right)}{\left[1+\lambda-\beta(1-\beta)(1-\alpha)^{2} m^{2}\right]^{2}} \\
& -\frac{2(1+\lambda) \beta(1-\alpha)(1-(1-\beta)(1-\alpha)) m}{\left[1+\lambda-\beta(1-\beta)(1-\alpha)^{2} m^{2}\right]^{2}}, \\
\frac{\partial \phi_{2}^{*}}{\partial m}= & -(1-\alpha) \psi \frac{(1-(1-\beta)(1-\alpha))\left(1+\lambda+\beta(1-\beta)(1-\alpha)^{2} m^{2}\right)}{\left[1+\lambda-\beta(1-\beta)(1-\alpha)^{2} m^{2}\right]^{2}} \\
& -\frac{2(1-\beta)(1-\alpha)(1+\lambda-\beta(1-\alpha)) m}{\left[1+\lambda-\beta(1-\beta)(1-\alpha)^{2} m^{2}\right]^{2}} .
\end{aligned}
$$

One can deduce from (14) and 15 that $\frac{\partial \phi_{1}^{*}}{\partial m}<0$ and $\frac{\partial \phi_{2}^{*}}{\partial m}<0$. The larger is $m$, the lower is the incentive to acquire knowledge of the native culture since interaction with natives becomes less probable. Finally, an increase of $\beta$ leads to asymmetric effects since it increases the population size of immigrants from country 2 which discourages this group from cultural learning. By contrast, cultural learning of immigrants from country 1 increases since matching with an immigrant from country 2 becomes more probable.

\footnotetext{
${ }^{4}$ Take into account that migrants may even achieve host country specific cultural skills larger then 1 . For migrants of source country 1 this is the case if $\psi>\frac{1+\lambda-\beta(1-\beta)(1-\alpha)^{2} m^{2}}{(1-\alpha)(1-m)(1+m(1-\beta)(1-\alpha))}$. Thus, if the migrant has sufficient career perspectives in the host economy, his knowledge of the host country's culture might be larger than his original.
} 


$$
\begin{aligned}
\frac{\partial \phi_{1}^{*}}{\partial \beta}= & (1-m) m \psi(1-\alpha)^{2} \frac{\lambda(1+(1-2 \beta)(1-\alpha) m)+1+m}{\left[1+\lambda-\beta(1-\beta)(1-\alpha)^{2} m^{2}\right]^{2}} \\
& -(1-m) m \psi(1-\alpha)^{2} \frac{2 \beta(1-\alpha) m+\beta^{2}(1-\alpha)^{2} m^{2}}{\left[1+\lambda-\beta(1-\beta)(1-\alpha)^{2} m^{2}\right]^{2}} \\
\frac{\partial \phi_{2}^{*}}{\partial \beta}= & (1-m) m \psi(1-\alpha)^{2} \frac{1+\lambda+2 \beta(1-\alpha) m}{\left[1+\lambda-\beta(1-\beta)(1-\alpha)^{2} m^{2}\right]^{2}} \\
& -(1-m) m \psi(1-\alpha)^{2} \frac{(1-\alpha) m\left(1+(1-\beta)^{2}(1-\alpha) m\right)}{\left[1+\lambda-\beta(1-\beta)(1-\alpha)^{2} m^{2}\right]^{2}}
\end{aligned}
$$

16) and 17 indicate that $\frac{\partial \phi_{1}^{*}}{\partial \beta}>0$ and $\frac{\partial \phi_{2}^{*}}{\partial \beta}<0$. The results are unambiguous since $m$ is assumed to be smaller than 0.5.

\section{$3 \quad$ Immigration Guidelines}

Natives can affect the cultural heterogeneity of migrants by use of a specific immigration policy. In this context, I assume that the scope of immigrants is exogenously determined. Suppose for example that immigration equals the number of required workers to fill a specific skill shortage or, more generally, to adjust to population shrinkage caused by the demographic change. Thus, I take the share of total migrants as predetermined and only derive the optimal composition of immigrants in order to study whether homogenous (immigration containing only one cultural group) or heterogeneous immigration is favorable. Assume that natives have an expected payoff function similar to the one of migrants, however, they do not bear any learning costs: $5^{5}$

$$
E W_{N}=\bar{w}\left(1-m+(1-\beta) m(1-\alpha) \phi_{1}+\beta m(1-\alpha) \phi_{2}\right)
$$

The native population can now decide whether the immigrant population should comprise only one or two subgroups. On the one hand, the native population could gain by a homogenous immigrant population which comprises only individuals from country 1 because their

\footnotetext{
${ }^{5}$ Since, we do not compare the income of migrants and natives, we do not have to make specific assumptions about the level of $\bar{w}$. One can of course assume that natives and immigrants on average have different qualification levels. This does not affect the analysis so that it can simply be ignored.
} 
learning costs are lower so that migrants in equilibrium will be better integrated than a mix of immigrants from country 1 and 2. However, (18) indicates that, if the total immigrant population comprises only individuals from country $1(\beta=0)$, the migrants' incentive to acquire cultural skills is low since the population size of this cultural group is relatively large. The latter argument would rather justify a heterogeneous immigrant population.

It is straightforward that if immigrants were purely symmetric $(\lambda=0)$ it would be optimal for the government to divide immigrants into two equally large subgroups by setting $\beta$ to 0.5 . In this scenario, the probability that a randomly chosen migrant matches with an individual of a different origin is the largest so that cultural learning is encouraged. However, if $\lambda>0$, the native population has to trade off the positive and negative effects of enlarged cultural heterogeneity among immigrants. At a certain level of $\lambda$, the difference between learning costs is so large that natives may only chose immigrants from country 1 .

Hence, it is reasonable to calculate the critical value for $\lambda$ where the government is exactly indifferent between choosing immigrants from one or two countries. This is the case when the welfare maximizing choice of $\beta$ is exactly 0 . Maximizing (18) with respect to $\beta$ leads to:

$$
\frac{d E W_{N}}{d \beta}=(1-\alpha) m\left(-\phi_{1}+(1-\beta) \frac{\partial \phi_{1}}{\partial \beta}+\phi_{2}+\beta \frac{\partial \phi_{2}}{\partial \beta}\right)=0 .
$$

Setting $\beta$ equal to 0 will give us the critical value for $\lambda$ where the government is indifferent between choosing immigrants from one or two countries:

$$
\widetilde{\lambda}=(1-\alpha) m(2+(1-\alpha) m) .
$$

If $\lambda<\widetilde{\lambda}$, the government will set $\beta>0$. This is illustrated by figure 2 . The expected native welfare will reach its maximum when $\beta$ is larger than 0 . It can be deduced from (20) that the choice for heterogeneous immigration is preferred if $m$ is large and if $\alpha$ and $\lambda$ are small. However, it is not influenced by $\psi$. It is clear that an increase of asymmetries in learning costs makes the government prefer immigrants from country 1 . The negative effect of enlarged cultural segregation on the integration decision of migrants is stronger if the community is smaller. This is true, since $\alpha$ is predominantly relevant if the random probability that a migrant matches with a migrant of the same cultural origin is relatively small. An increase in the entire scope of migrants decreases cultural learning of all migrants. This is mainly the case if migrants are homogenous. Increased cultural heterogeneity of migrants therefore constitutes an opportunity to counteract this effect. 


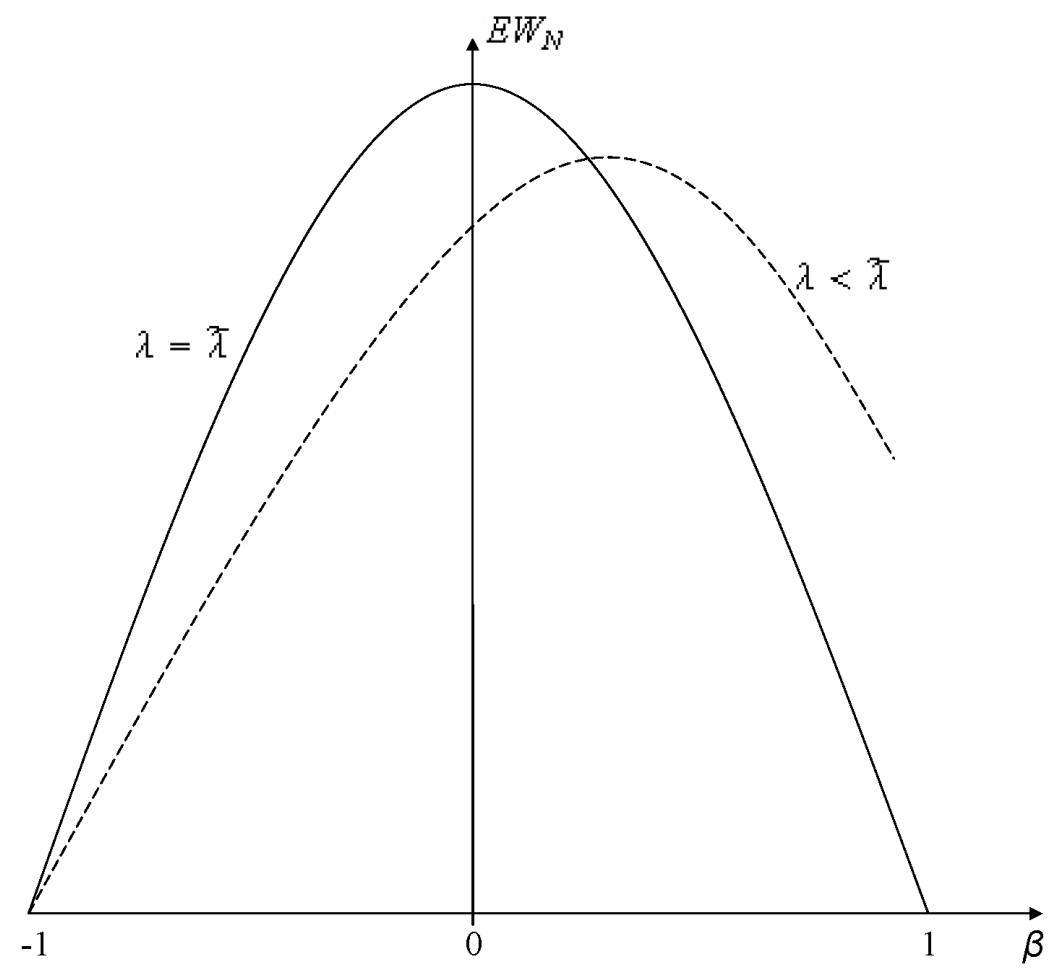

Figure 2: Optimal value of beta depending on the integration cost asymmetries between country 1 and 2 . 


\section{Intra- vs. Inter-Group Heterogeneity}

Until now, it has been assumed that the government can choose between immigrants from two countries whose members are homogenous and can communicate and interact with other members of the same origin without obstacles. Let us now extend the framework by assuming that individual migrants are divided into two country groups with different integration costs. For example, have in mind that a country may preferentially permit immigration from all neighborhood countries, members of a federal union like the EU or former colonies whose populations share lower costs of learning the host country's culture. However, these countries are also characterized by different cultures, namely by different languages, religions or cultural values. Moreover, the government can allow immigration from the rest of the world as a second potential country group to even further increase the level of cultural heterogeneity among the immigrant population.

In this modified framework, one can thus distinguish intra-group heterogeneity with intergroup heterogeneity. It is reasonable to suppose that increased intra-group heterogeneity may reduce the necessity to discipline immigrants from the cultural affine country group 1 by permitting additional immigration from country group 2. The framework of the former section changes in the way that migrants can now additionally interact with migrants of different origins who have identical learning costs. Imagine for example that Germans and French people have the same costs of learning the English language when they settle in Great Britain.

For simplicity, suppose that both country groups comprise the same number $z$ of equally large countries. Thus if a migrant matches with a migrant from the same country group,

the probability is $\rho=\frac{z-1}{z}$ that the migrants are of different cultural origin. By limiting the number of country groups to 2, one achieves the following expected payoff function for a representative agent from country group 1 and 2 :

$$
\begin{aligned}
& E W_{M_{1, h}}= \bar{w}\left[(1-m)(1-\alpha) \psi \phi_{1, h}+\alpha+(1-\alpha) \rho(1-\beta) m\right. \\
&\left.+(1-\rho)(1-\beta)(1-\alpha) m \phi_{1, h} \phi_{1, g \neq h}+\beta m(1-\alpha) \phi_{1, h} \phi_{2}-0.5 \cdot \theta_{1, h}^{2}\right],(21) \\
& E W_{M_{2, k}}=\bar{w}\left[(1-m)(1-\alpha) \psi \phi_{2, g}+\alpha+(1-\alpha) \rho \beta m+(1-\rho) \beta(1-\alpha) m \phi_{1, k} \phi_{1, l \neq k}\right. \\
&+ \\
&\left.+(1-\beta) m(1-\alpha) \phi_{2, k} \phi_{1}-(1+\lambda) 0.5 \cdot \theta_{2, k}^{2}\right]
\end{aligned}
$$


where index $1, h$ indicates that the individual comes from country $h$ which belongs to country group 1 and 2, $k$ describes an individual from country $k$ in country group 2. Maximizing $E W_{M_{i, h}}$ with respect to $\phi_{i, h}$ leads to:

$$
\begin{aligned}
\phi_{1, h} & =(1-\alpha)(1-m) \psi+(1-\alpha) \beta m \phi_{2}+(1-\alpha) \rho(1-\beta) m \phi_{1, g \neq h}, \\
\phi_{2, h} & =\frac{(1-\alpha)(1-m) \psi+(1-\alpha) \beta m \phi_{1}+(1-\alpha) \rho(1-\beta) m \phi_{2, g \neq h}}{1+\lambda} .
\end{aligned}
$$

Since all immigrant populations are of the same size and learning costs within country groups are equal, one can apply symmetry and conclude that $\phi_{i, h}=\phi_{i, g \neq h}=\phi_{i}$. Hence, the cultural learning intensity of immigrants is:

$$
\begin{gathered}
\phi_{1}=\frac{(1-\alpha)\left((1-m) \psi+\beta m \phi_{2}\right)}{1-(1-\alpha) \rho(1-\beta) m}, \\
\phi_{2}=\frac{(1-\alpha)\left((1-m) \psi+(1-\beta) m \phi_{1}\right)}{1+\lambda-(1-\alpha) \rho \beta m} .
\end{gathered}
$$

The intersection point of (25) and (26) gives us the optimal values for language acquisition of migrants from country group 1 and 2.

$$
\begin{aligned}
\phi_{1}^{*} & =\frac{(1-\alpha)(1-m) \psi(1+\lambda+(1-\alpha) \beta m(1-\rho))}{(1-(1-\alpha) \rho(1-\beta) m)(1+\lambda-(1-\alpha) \rho \beta m)-m^{2} \beta(1-\beta)(1-\alpha)^{2}} \\
\phi_{2}^{*} & =\frac{(1-\alpha)(1-m) \psi(1+(1-\alpha)(1-\beta) m(1-\rho))}{(1-(1-\alpha) \rho(1-\beta) m)(1+\lambda-(1-\alpha) \rho \beta m)-m^{2} \beta(1-\beta)(1-\alpha)^{2}}
\end{aligned}
$$

The equilibrium levels indicate that increasing heterogeneity within each country group improves cultural learning of immigrants from both country groups. A proof is provided in the Appendix. This is intuitive since a larger $\rho$ decreases the probability that immigrants interact with individuals of the same cultural origin. Furthermore, the results illustrate that if country groups are sufficiently diverse with regard to their member countries, heterogeneity among country groups is not useful but may even be harmful with respect to the migrants' learning ambition. In the extreme case of an infinite number of cultures within each country groups $(\rho \rightarrow 1), 227)$ and $(28)$ change to:

$$
\lim _{\rho \rightarrow 1} \phi_{1}^{*}=\frac{(1-\alpha)(1-m) \psi(1+\lambda)}{(1-(1-\alpha) m)(1+\lambda)+\beta \lambda(1-\alpha) m},
$$




$$
\lim _{\rho \rightarrow 1} \phi_{2}^{*}=\frac{(1-\alpha)(1-m) \psi}{(1-(1-\alpha) m)(1+\lambda)+\beta \lambda(1-\alpha) m} .
$$

In this case, cultural learning of all migrants depends negatively on the share of immigrants from country group 2. The result is straightforward. If heterogeneity within each country group is already extremely large, the members of country group 1 do not need to be additionally disciplined by increased heterogeneity. Yet, this would even decrease their ambition since the individuals who are comprised by country group 2 have higher learning costs. Due to the positive externality between the learning ambitions of migrants from country group 1 and 2 , all individuals benefit from lower learning costs of the other migrants. Thus, interaction of migrants from the same group may increase the learning ambition of migrants.

Furthermore, it is useful to analyze the expected utility that is gained by the native population which is still captured by (18), yet, in this case, the indices 1 and 2 do not

describe countries but country groups. Maximizing (18) with respect to $\beta$ thus leads to (19) Setting $\beta$ equal to 0 will give us the critical value for $\lambda$ where the government is indifferent between choosing immigrants from one or two country groups:

$$
\widetilde{\lambda}_{c g}=(1-\alpha) m(1-\rho)(2+(1-\alpha) m(1-\rho))
$$

Equation (31) is of course a general case of the basic model. Thus, it is not surprising that the effects of the former section with regard to $\alpha, m$ and $\psi$ do not change qualitatively. However, one can deduce that an increase in intra-group heterogeneity $\rho$ reduces the threshold level of learning cost asymmetries $\widetilde{\lambda}_{c g}$. This in turn implies that at a given rate of learning cost asymmetries, increased intra-group heterogeneity diminishes the need for inter-group heterogeneity. If the number of countries within each cultural group is infinitely large $(\rho \rightarrow 1)$, the government concentrates on immigrants from country group 1.

\section{Cultural Selection in Immigration Policies}

The model describes some aspects which are significant for the immigration guidelines of many countries throughout the 20th century. In this section, I try to employ the results of the theoretical model to explain why the traditional settlement countries Australia, Canada, New Zealand and the U.S. redesigned their immigration policies in the 1970s. I also point at cross country differences between the European Union and the Anglo-Saxon states.

Immigration policies that restricted immigration to applicants who shared a common 
culture with the native population have for long been in place in many countries. At the beginning of the 20th century, due to a supposed rising threat to the dominant Anglo-Saxon culture, the U.S. implemented a series of restrictions that excluded potential immigrants from China and other Asian countries (Hatton and Williamson 2005). Similar developments have taken place in the other traditional immigration countries (Jupp 1998). The Australian Immigration Restriction Act of 1901 was resigned to ensure a White Australia Policy. The labor scarce countries, however, required immigrants who were predominantly attracted from Southern and Eastern European countries. By this, it was anticipated that assimilation into the native society would proceed more rapidly. Ethnic origin had in fact been the key variable which managed immigration until the early 1970s when these discriminatory laws were replaced by a multicultural immigration policy (Leuner 2007).

One explanation for this shift in immigration policy was probably that cost asymmetries between different source countries had declined over time. As shown by the theoretical model, low learning cost asymmetries support an immigration policy that promotes cultural diversity. This mainly happened due to two mutually enhancing developments. Technological progress has reduced information and communication costs and thus reduced information asymmetries around the world. The possibility to acquire foreign language skills for instance has improved significantly over time. At the same time, the political and economic transformations of many countries like China and Russia have lead to a sharp increase in international trade and global capital flows. Accordingly, overall cultural knowledge should have increased over time, diminishing the importance of geographical and cultural distance.

Another important issue refers to the scope of the entire migrant population. The theoretical model predicted that increases in the overall scope of migrants discourage the government from conducting a selective immigration policy. In order to prevent the upcoming of one large but poorly integrated minority culture, the government has incentives to foster multiculturalism among the foreign born population. In fact, the share of foreign born residents have increased in Australia, Canada and New Zealand from around 15\% in 1960 to somewhat 21\% in 2010. In the U.S. it increased from 6\% in 1960 to almost 14\% in 2010 (World Bank 2012). Hence, the shift in immigration policy might also be explained by an increase in the entire scope of the migrant population.

A similar development has been taking place in the European Union member states. However, compared to the traditional settlement countries of the former British empire, the individual European cultures are much less spread around the world. Again language skills might serve as an adequate example. Over the time, English has probably become the most relevant second language. Its impact on international exchange has by far outreached the im- 
portance of other European languages. Hence, cultural and linguistic distance might still play a much larger role in Continental Europe than in the Anglo-Saxon countries. Furthermore, differences with regard to the overall foreign born population have only slightly narrowed over time. Even though, the stock of the foreign born population has more than doubled in the EU from 1960 to 2010, it is still below the 10\% level and thus not comparable at least with the degrees in Australia, Canada and New Zealand (World Bank 2012).

Additionally, there has been another series of developments which are predominantly important for European countries and may also to some extent drive the immigration policies within the member states of the European Union. The collapse of the Soviet Union has lead to a tremendous resurgence of cultural diversity on the European continent since the new independent countries revitalized their cultural identities which had often been repressed by the former central government. A similar development has taken place in former Yugoslavia and Czechoslovakia. Up to now, there is a tendency of countries rather decomposing than unifying ${ }^{6}$ and cultural minorities as the Basques or Catalans in Spain or the Walloons in Belgium have won a share of political autonomy on the basis of their national identity (Castells 2011).

Moreover, due to the political liberalization of many countries within the last decades, the number of potential source countries has increased significantly over time. To some extent, this may explain why European governments tend to allow immigration from culturally related countries within the European Union but mostly restrict entrance from non European source countries. Potentially, intra-group heterogeneity within the European Union is sufficiently large so that immigrants from the rest of the world are not required or even harm an integration process of migrants.

\section{Conclusions}

This paper has employed a simple theoretical model to analyze how cultural heterogeneity of migrants affects their ambition to acquire host country specific cultural skills. The model shows that enlarged cultural diversity ceteris paribus increases the migrants' incentive to learn the native culture. This argument is centered around the empirical fact that cultural learning decreases in the relative population size of the respective cultural group. Furthermore, cultural learning of migrants is encouraged if the structure of the host country counteracts cultural segregation and improves the career perspectives of immigrants.

\footnotetext{
${ }^{6}$ One can think of Sudan as a current example. Furthermore there are tendencies towards disintegration in Great Britain, Sri Lanka, India, Indonesia and Nigeria (Castles 2011).
} 
With regard to immigration policy, the model suggests that a government can maximize the native welfare by choosing a level of cultural heterogeneity among the migrant population. If the potential source countries are not too heterogeneous with respect to learning costs, the government is likely to be in favor of a multicultural immigration policy which avoids that minority cultures become large and thus influential. If integration costs are sufficiently asymmetric, a discriminatory selective immigration policy focusing on immigrants from culturally close countries is optimal for a government that aims at maximizing the welfare of the native population.

The paper also distinguished between intra-group and inter-group heterogeneity. The results of the theoretical model predicted that enlarged intra-group heterogeneity can be a substitute for inter-group heterogeneity and can thus support a discriminatory immigration policy for the culturally close country group. To some extent, the model explains why immigration policies have changed over time and why they still differ across host countries. In this context, I mainly focused on the importance of learning costs asymmetries between migrants, the scope of the migrant population as well as intra-group heterogeneity as the main determinants of these developments. I conclude that increased intra-group heterogeneity reduces the necessity to attract workers from culturally distant countries, whereas a reduction of learning cost asymmetries between applicants from different source countries and an increase in the relative population size of overall migrants have made policy makers to be in favor of multicultural immigration policies.

\section{References}

[1] Bonn, M. (2012), "Costs and benefits from Immigration and multicultural interaction," MAGKS Working Paper 40.

[2] Burchardi, K. B. (2011), "Language heterogeneity and structural change," IIES Working Paper.

[3] Castles, M. (2011), "The information age: Economy, society and culture volume II: The power of identity," 2nd edition, Oxford: Blackwell.

[4] Chaloff, J. and G. Lemaitre (2009), "Managing highly skilled labour migration: A comparative analysis of migration policies and challenges in OECD countries," OECD Social, Employment and Migration Working Paper 79. 
[5] Chiswick, B. R. (1978), "The effect of americanization on the earnings of foreign-born men," Journal of Political Economy, 86: 897-921.

[6] Chiswick, B. R. and P. W. Miller (1995), "The endogeneity between language and earnings: International analyses," Journal of Labor Economics, 13 (2): 246-288.

[7] Chiswick, B. R. and P. W. Miller (1998), "English language fluency among immigrants in the United States," Research in Labor Economics, 17: 151-200.

[8] Chiswick, B. R. and P. W. Miller (1999), "Language skills and earnings among legalized aliens," Journal of Population Economics, 12 (1): 63-89.

[9] Chiswick, B. R. and P. W. Miller (2002), "Immigrant earnings: language skills, linguistic concentrations and the business cycle," Journal of Population Economics, 15: 31-57.

[10] Church, J. and I. King (1993), "Bilingualism and network externalities," Canadian Journal of Economics, 26 (2): 337-345.

[11] Green, A. G. (1995), "A comparison of Canadian and U.S. immigration policy in the twentieth century," in Devoretz, D. J. (ed.), "Diminishing returns: The economics of Canada's recent immigration policy," Toronto: Laurier: 31-64.

[12] Hatton, T. J. and J. G. Williamson (2005), "Global migration and the world economy. Two centuries of policy and performance," Cambridge, MA: MIT Press.

[13] Hugo, G. (2011), "Future demographic change and its interactions with migration and climate change," Global Environmental Change, 21: S21-S33.

[14] Jupp, J. (1998), "Immigration," Melbourne: Oxford University Press.

[15] Lazear, E. P. (1999), "Culture and language," Journal of Political Economy, 107: 95-126.

[16] Leuner, B. (2007), "Migration, multiculturalism and language maintenance in Australia: Polish migration to Melbourne in the 1980s," Bern: Peter Lang Publishing.

[17] United Nations (2009), "World population prospects: The 2008 revision," New York: United Nations.

[18] The World Bank (2012), "World Development Indicators (WDI) and Global Development Finance (GDF)," http://databank.worldbank.org/ddp/home.do (accessed on 05.10.2012). 


\section{A Appendix}

\section{A.1 Cultural diversity within a cultural group and integration of migrants}

Proposition 1 An increase of cultural heterogeneity within each country group expressed by $\rho$ increases equilibrium integration of migrants expressed by $\phi_{1}^{*}$ and $\phi_{2}^{*}$.

Proof. From (25) and (26) one can deduce that

$$
\begin{aligned}
& \phi_{1}^{*}=A+B\left(C+D \phi_{1}^{*}\right), \\
& \phi_{2}^{*}=C+D\left(A+B \phi_{2}^{*}\right) .
\end{aligned}
$$

where $A=\frac{(1-\alpha)(1-m) \psi}{1-(1-\alpha) \rho(1-\beta) m}, B=\frac{(1-\alpha) \beta m}{1-(1-\alpha) \rho(1-\beta) m}, C=\frac{(1-\alpha)(1-m) \psi}{1+\lambda-(1-\alpha) \rho \beta m}$ and $D=\frac{(1-\alpha)(1-\beta) m}{1+\lambda-(1-\alpha) \rho \beta m}$. Partial differentiating with respect to $\rho$ leads to:

$$
\begin{aligned}
\frac{\partial \phi_{1}^{*}}{\partial \rho} & =\frac{\frac{\partial A}{\partial \rho}+\frac{\partial B}{\partial \rho}\left(C+D \phi_{1}^{*}\right)+B\left(\frac{\partial C}{\partial \rho}+\phi_{1}^{*} \frac{\partial D}{\partial \rho}\right)}{1-B D} \\
\frac{\partial \phi_{2}^{*}}{\partial \rho} & =\frac{\frac{\partial C}{\partial \rho}+\frac{\partial D}{\partial \rho}\left(A+B \phi_{2}^{*}\right)+D\left(\frac{\partial A}{\partial \rho}+\phi_{2}^{*} \frac{\partial B}{\partial \rho}\right)}{1-B D} .
\end{aligned}
$$

Since $\frac{\partial A}{\partial \rho}>0, \frac{\partial B}{\partial \rho}>0, \frac{\partial C}{\partial \rho}>0, \frac{\partial D}{\partial \rho}>0$, it is clear that the numerators of $(34)$ and $(35)$ are positive. The denominators are positive since

$$
\begin{aligned}
1-B D= & \frac{(1-(1-\alpha) m)+\lambda(1-(1-\alpha) \rho(1-\beta) m)}{1-(1-\alpha) \rho(1-\beta) m} \\
& +\frac{(1-\alpha)(1-\rho) m(1-(1-\alpha)(1+\rho) \beta(1-\beta) m)}{1-(1-\alpha) \rho(1-\beta) m} .
\end{aligned}
$$

